\title{
Robust Gait Control in Biomimetic Amphibious Robot Using Central Pattern Generator
}

\author{
Rui Ding, Junzhi Yu, Qinghai Yang, Min Tan, and Jianwei Zhang
}

\begin{abstract}
This paper presents a control architecture for the underwater locomotion control of a biomimetic amphibious robot with multi-mobility mechanism. In view of both hydrodynamic problem and engineering approach, we develop a robotic prototype capable of multi-mode motion. A robust gait control for steady swimming using the central pattern generator (CPG) is proposed and has been successfully applied to the robot. The CPG can produce coordinated patterns of rhythmic activity while being simply modulated by control parameters including input drive, frequency, amplitude, threshold, etc., which will be suitable for manually interactive modulation. Using the CPG model, the robot is capable of performing and switching between various locomotion modes such as swimming forwards and backwards, turning and pitching, with the speed, direction and gait types modulated accordingly. A test-bed is provided and results are presented demonstrating interesting properties of the CPG-based control approach and feasibility of the CPG control for efficient propulsion.

Index Terms-Amphibious robot, hydrodynamic, gait control, central pattern generator, multi-mode motion.
\end{abstract}

\section{INTRODUNCTION}

Fish in nature have skyscraping underwater thrust efficiency, which outperforms the conventional screw propeller currently in use. Of recent interest has been the research of biomimetic robotic fish, which has created a new realm of biomimetic robots. In order to mimic the oscillation property of the fish body, Lighthill's elongated body theory has been widely adopted to generate propulsive traveling wave [1]. Many other aspects of fish-swimming behavior have been studied to shed light on the propulsion mechanism [2]-[4], which demonstrate implied undulatory wave traveling from head to tail periodically with increasing amplitude.

Meanwhile neurobiology studies reveal that fundamental rhythmic motor patterns in locomotion are generated by central pattern generators $(C P G s)$ in various organisms. The CPGs are networks of neurons that can produce coordinated oscillatory signals without oscillatory inputs from sensory feedback or from higher control centers, which are widely used in robotics recently [5]-[7]. A typical example of CPG is found in the lamprey and has been studied extensively [8].

This work was partly supported by the National Natural Science Foundation of China $(60775053,60865004)$, the National 863 Program (2007AA04Z202), and the Beijing Natural Science Foundation (4082031, 4102063).

R. Ding, J. Yu, Q. Yang, and M. Tan are with the Key Laboratory of Complex Systems and Intelligence Science, Institute of Automation, Chinese Academy of Sciences, Beijing 100190, China. Currently J. Yu is also a guest researcher supported by the Alexander von Humboldt Foundation with the Department of Informatics, University of Hamburg, Germany. rui.ding@ia.ac.cn

J. Zhang is with the Department of Informatics, University of Hamburg, Germany.

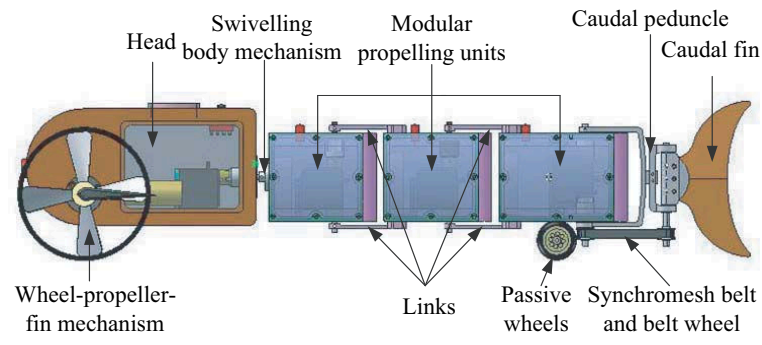

Fig. 1. The mechanism configuration of the AmphiRobot.

The CPG has also representatively been applied to the salamander robot for walking and swimming locomotion [9].

In this paper, the CPG model is applied to a multi-mode biomimetic amphibious robot for underwater locomotion control. The amphibious robot, AmphiRobot, as shown in Fig. 1, is composed of a head, alternative wheel-propellerfin mechanism, swivelling body mechanism, modular propelling units, peduncle and caudal fin. The AmphiRobot draws inspiration of wheeled mobile robot on land and undulatory body motions of robotic fish in water. On land, the AmphiRobot utilizes a two-wheeled structure to propel itself to crawl forward while pectoral fins coordinated with modular fish-like propelling units can achieve efficient and agile swimming performance in water. A specially designed swivelling body mechanism can implement intelligent switch between the fish-swimming mode and dolphin-swimming mode.

The AmphiRobot takes fish-like swimming as primary underwater motor pattern, by utilizing the traveling waves of body undulation transmitted form head to tail for propulsion. Thus, CPGs can also be used to produce the waves of joint activation for fish-like swimming of the AmphiRobot. One interesting aspect of this approach is that the CPG model has explicit parameters defining quantities such as frequency, amplitude, input drive, and threshold, which can be continuously and interactively modulated by a human operator. We thus obtain a control architecture in which various locomotor gaits can be generated with the speed and direction modulated accordingly.

The rest of the paper is organized as follows. In Section II, an overall description of the hydrodynamic model and amphibious robotic prototype are provided. Section III describes the CPG model and corresponding control parameters in detail. Based on the proposed CPG model, the simulated and experimental results are presented in Section IV. Section V 
concludes the paper with the summary about the current work and an outline of future work.

\section{Development of Amphibious Robot}

\section{A. Hydrodynamic Model of Amphibious Robot}

The modular designed amphibious robot can be regarded as a multi-link serial mechanism coupling of pectoral fins. From the perspective of rigid body dynamics, the AmphiRobot will be simplified as a five-link serial mechanism coupled with simplified pectoral fins, as shown in Fig. 2. Thereamong, the first link corresponds to the head connected by the first modular propelling unit and the last to the caudal fin.

Denote the potential energy as a constant $E$. The kinetic energy of each oscillating element is comprised of translational kinetic energy under the world rectangular coordinate system (WRCS) and the rotational kinetic energy under the center-of-mass system. The coordinate system (WRCS) $X O Y$ is established with the origin placed at the starting point of the second link and the locomotion is limited in the horizontal plane, so the Lagrangian function will be expressed as follows:

$$
\begin{aligned}
L= & \sum_{i=1}^{N} \frac{1}{2} m_{i} v_{i}^{2}+\sum_{i=1}^{N} \frac{1}{2} I_{i} w_{i}^{2}+\frac{1}{2} m_{L} v_{L}^{2}+\frac{1}{2} I_{L} w_{L}^{2} \\
& +\frac{1}{2} m_{R} v_{R}^{2}+\frac{1}{2} I_{R} w_{R}^{2}-E \\
= & \sum_{i=1}^{N} \frac{1}{2} m_{i}\left(\left(\dot{x}_{i}^{g}\right)^{2}+\left(\dot{y}_{i}^{g}\right)^{2}\right)+\sum_{i=1}^{N} \frac{1}{2} I_{i}\left(\dot{\beta}_{i}\right)^{2} \\
& +\frac{1}{2} m_{L}\left(\left(\dot{x}_{L}^{g}\right)^{2}+\left(\dot{y}_{L}^{g}\right)^{2}\right)+\frac{1}{2} I_{L}\left(\dot{\varphi}_{L}\right)^{2} \\
& +\frac{1}{2} m_{R}\left(\left(\dot{x}_{R}^{g}\right)^{2}+\left(\dot{y}_{R}^{g}\right)^{2}\right)+\frac{1}{2} I_{R}\left(\dot{\varphi}_{R}\right)^{2}-E
\end{aligned}
$$

where $m_{i}$ is the mass of the $i$ th link, $I_{i}$ is the estimated moment of inertia of the $i$ th link under the center-of-mass system, $m_{L}$ is the mass of the left pectoral fin, and $I_{L}$ is its estimated moment of inertia. $\left(x_{i-1}, y_{i-1}\right)$ and $\left(x_{i}, y_{i}\right)$ are individually the starting point and end point of the $i$ th link. $\left(x_{i}^{g}, y_{i}^{g}\right)$ and $\left(x_{i}^{f}, y_{i}^{f}\right)$ are the barycenter and the centroid (center of figure) of the $i$ th link separately. $\left(x_{L}^{g}, y_{L}^{g}\right)$ and $\left(x_{L}^{f}, y_{L}^{f}\right)$ are the barycenter and centroid of the left pectoral fin separately. $\beta_{i}$ denotes the angle between the $i$ th link and the $X$-axis $(i=1, \ldots, 5)$. Analogously, $\varphi_{i}$ represents the angle between the $(i+1)$ th link and the extension line of the $i$ th link $(i=1, \ldots, 4)$, that is, the actuated joint angle signal of the $(i+1)$ th link. $\varphi_{L}$ is the angle between the left pectoral fin and the horizontal plane, while $\varphi_{R}$ for the right pectoral fin. All joint angles are anti-clockwise positive. The symbols for the right pectoral fins are just empathetic. $x_{1}, y_{1}$ and $\beta_{2}$ are selected as the generalized coordinates, and let $X=x_{1}$, $Y=y_{1}, \Theta=\beta_{2}$, then the Lagrange's equations of the second kind can be given by

$$
\left\{\begin{array}{l}
F_{X}=\frac{d}{d t} \frac{\partial L}{\partial X}-\frac{\partial L}{\partial X} \\
F_{Y}=\frac{d}{d t} \frac{\partial L}{\partial \dot{Y}}-\frac{\partial L}{\partial Y} \\
M_{\Theta}=\frac{d}{d t} \frac{\partial L}{\partial \Theta}-\frac{\partial L}{\partial \Theta}
\end{array}\right.
$$

where $F_{X}$ and $F_{Y}$ are the compositions of the hydrodynamic forces on the $X$ axis and $Y$ axis, respectively. $M_{\Theta}$ is the moment relative to the reference point $(X, Y)$ during locomotion.

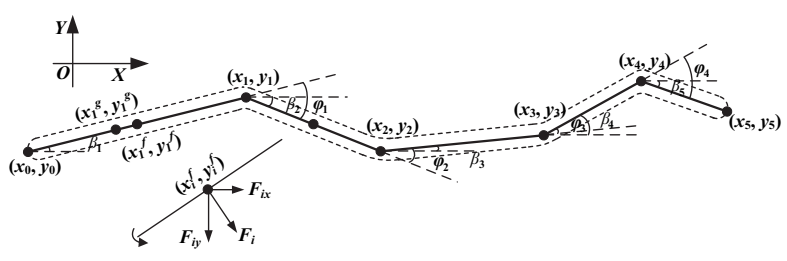

(a)

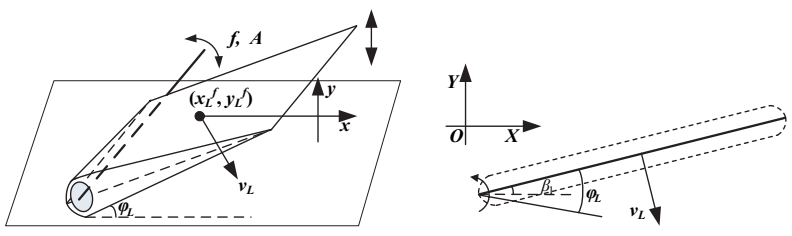

(b)

Fig. 2. Simplified mechanical structure of the AmphiRobot. (a) Five-link serial mechanism. (b) Simplified pectoral fin.

Robot swimming underwater interacting with the fluid flow remains an unresolved problem. In many cases, some simplifications are necessary. To calculate the resultant forces, a large Reynolds number is applied and all forces acting on a propulsive element are due to the motion of that element in the fluid, i.e., the effects of the fluid's motion are not considered. The resultant hydrodynamic force perpendicular to the surface of the moving body takes the form:

$$
F=-\mu v|v|,
$$

where $\mu=\rho C S / 2$ is the drag coefficient, $\rho$ is the density of the fluid, $C$ is the shape-dependent drag coefficient, $S$ is the effective area of the element that confronts the fluid, and $v$ is the speed of the element.

The robot is thus acted upon by four types of forces: pressure on links, pressure on pectoral fins, approach stream pressure, and friction drag. We can resolve these forces into components which act parallel and perpendicular to the surface of each element. By resolving all the forces acting on the oscillating elements in the WRCS, a system of associated equations of motion on the basis of $X, Y$, and $\Theta$ can be obtained. A dynamic simulation of the amphibious robot swimming can ultimately be implemented in Mathematica environment.

\section{B. Development of Robotic Prototype}

Fig. 3 shows the developed amphibious prototype with technical parameters listed in Table I. For the purpose of drag reduction, convex camber shells for fish-like modules are utilized to form a well-streamlined body which will offer less resistance to fluid flow. In order to active freely in both underwater and land environments, some sensors are mounted to perceive the external environment. The control architecture of the amphibious robot is then established based on an ARM centered embedded control system [10], in which control algorithms can be programmed onboard and highlevel commands can be continuously received wirelessly via an antenna to modulate the locomotion speed and direction. 


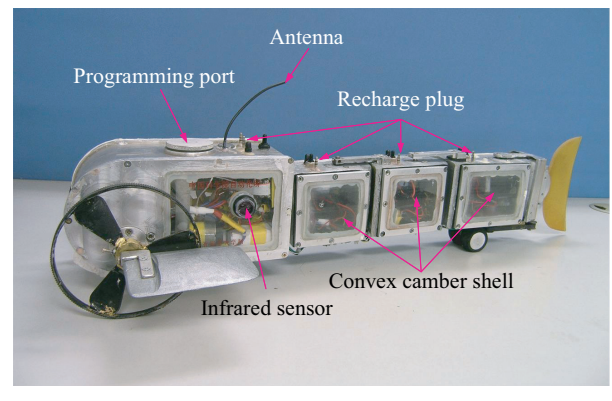

Fig. 3. The prototype of the developed AmphiRobot.

TABLE I

TECHNICAL PARAMETERS OF THE DEVELOPED AMPHIROBOT

\begin{tabular}{ll}
\hline Terms & Characteristics \\
\hline Dimension $(L \times W \times H)(\mathrm{mm})$ & $\sim 700 \times 320 \times 150$ \\
Total mass $(\mathrm{kg})$ & $\sim 4.995$ \\
& 3 infrared sensors +1 pressure sensor \\
Sensors & +2 liquid-level sensors \\
Maximum oscillating frequency $(\mathrm{Hz})$ & 3.3 (for pectoral fin in calm water) \\
Maximum servomotor torque $(\mathrm{kg} \cdot \mathrm{cm})$ & 30 (at $7.4 \mathrm{~V})$ \\
Actuator mode & $2 \mathrm{DC}$ motors +7 servomotors \\
Operation time & $\sim 1 \mathrm{~h}$ \\
Control mode & $\mathrm{RF}(433 \mathrm{MHz})$ or autonomous mode \\
\hline
\end{tabular}

\section{CPG-BASED LOCOMOTION CONTROL}

\section{A. Neural Oscillator Model}

Neural oscillator is the basic cell of the CPG network. Taking into account the capital property that CPGs are neural networks capable of producing coordinated patterns of rhythmic activity, differential equation is generally used to describe the neural oscillator. The adopted oscillator model is described by the following equations [9]:

$$
\left\{\begin{array}{l}
\dot{\theta}_{i}=2 \pi f_{i}+\sum_{j \in T(i)} a_{j} w_{i j} \sin \left(\theta_{j}-\theta_{i}-\phi_{i j}\right) \\
\ddot{a}_{i}=\tau_{i}\left\{\frac{\tau_{i}}{4}\left(A_{i}-a_{i}\right)-\dot{a}_{i}\right\} \\
x_{i}=a_{i}\left\{1+\cos \left(\theta_{i}\right)\right\}
\end{array}\right.
$$

where $\theta_{i}$ and $a_{i}$ represent the state variables, respectively, the phase and amplitude of oscillator $i, f_{i}$ and $A_{i}$ determine its intrinsic frequency and amplitude, and $\tau_{i}$ is a positive constant determining the speed $a_{i}$ converges to $A_{i}$. Couplings between oscillators are defined by the coupling weights $w_{i j}$ and phase biases $\phi_{i j}$. A positive oscillatory signal $x_{i}$ for oscillator $i$ represents the resultant burst serving as the output signal. $T(i)$ is the discrete set of oscillators which inflict inbound couplings with oscillator $i$.

In the above equations, the undetermined parameters include $f_{i}, A_{i}, w_{i j}, \phi_{i j}$ and $\tau_{i} . \tau_{i}$ only affects the time generating the oscillation signal, which is insignificant to the waveform and can be set previously. $f_{i}$ and $A_{i}$ indicate the behavior property of a single oscillator while $w_{i j}$ and $\phi_{i j}$ determine the connections among oscillators, ultimately the structure of the CPG network. Given these parameters, the oscillation signals of oscillators are consequently generated, and then the behavior properties of the CPG network are determined.

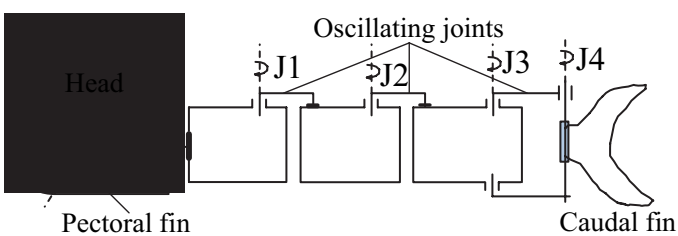

Fig. 4. The kinematical diagram of the AmphiRobot.

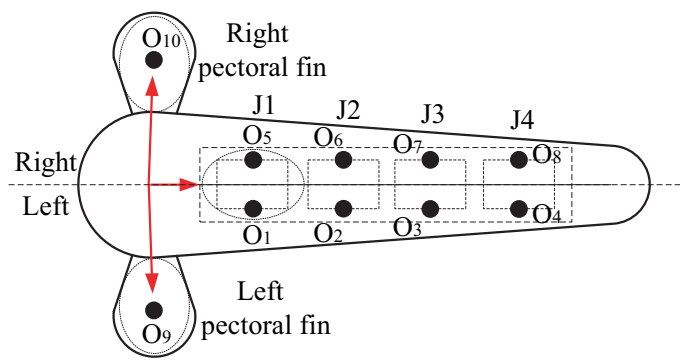

Fig. 5. The chain-like CPG network of the AmphiRobot.

Using the oscillator model, periodic oscillatory signals can be produced, which can be utilized as the joint actuation signals for undulatory mechanism. The CPG model for the AmphiRobot is then regarded as the coupling and mutual connections among oscillators in the oscillating elements.

\section{B. CPG Network}

The commonly used CPG network can typically fall into two categories: chain structure and network structure. In the chain CPG, rhythmic signals are transmitted from one side to another with a certain phase lag among oscillators. The AmphiRobot takes fish-like swimming as the primary underwater motor pattern. During swimming, several fishlike oscillating hinge joints (J1-J4) comprise the drive mechanism while coordinated with a pair of pectoral fins located in the head symmetrically, as shown in Fig. 4. According to the body structure of the AmphiRobot, it conforms to the chain-like CPG, thus the chain-like CPG model can be applied to produce coordinated rhythmic patterns, i.e. traveling waves for propulsion.

The CPG network for the AmphiRobot is composed of pectoral CPG and tail CPG, as illustrated in Fig. 5. The pectoral fin on each side corresponds to a nonlinear oscillator, $O_{9}$ and $O_{10}$ separately, with the output signal as the control command to drive the pectoral fin. The actuated joint angle signals of pectoral fins can be expressed as $\varphi_{L}=x_{9}-a_{9}$, $\varphi_{R}=x_{10}-a_{10}$, which have been modulated to be positivenegative. $L$ and $R$ are the abbreviations representing the left and right sides of the robot. The tail CPG is made up of eight oscillators, i.e. $O_{1}-O_{8}$, every two of which constitute a CPG unit for each oscillating joint (J1-J4), corresponding to a pair of mutually inhibited extensor and flexor. The subtraction of output of the left and right body oscillators in each oscillating joint is utilized to actuate the homologous joint, that is, $\varphi_{i}=x_{i+4}-x_{i}(i=1, \ldots, 4)$.

Fish swimming underwater should be agile to modulate the speed to finely adapt gaits to the external environmental 


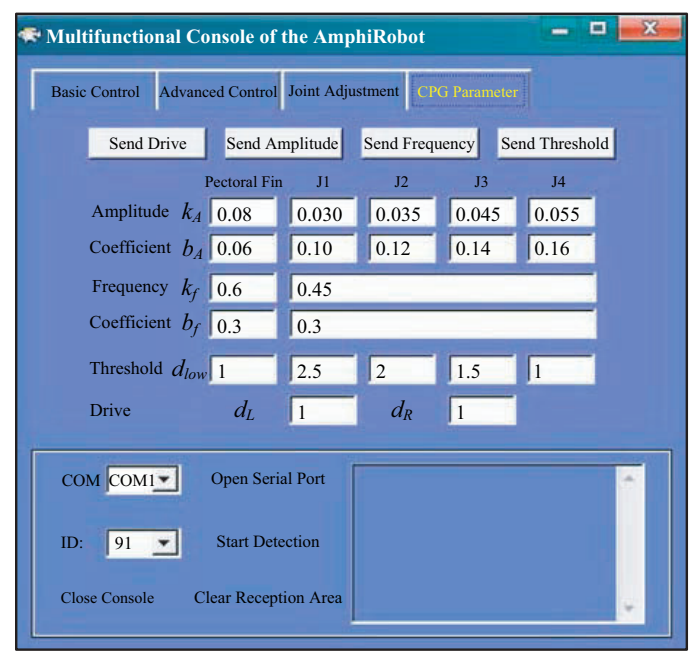

Fig. 6. The control platform of the AmphiRobot.

conditions. In general, fish achieve the speed adjustment by changing the oscillation frequency and amplitude of oscillating joints, which can be achieved by modulating the intrinsic frequency $f_{i}$ and amplitude $A_{i}$ according to (3). The swimming mechanism of fish in nature remains a crucial unresolved problem. It is difficult to find a conclusion on how the locomotion speed depends on the parameters $f_{i}$ and $A_{i}$ for different activities, e.g., steady swimming, rapid starts, escape maneuvers, turns, etc. Incorporating the engineering suggested by a live biological fish into a robot capable of autonomous free swimming and maneuvering underwater is a daunting and cumbersome task. For simplicity, linear functions are adopted here, that is

$$
\begin{gathered}
f_{i}=g_{f}(d)= \begin{cases}k_{f, i} d+b_{f, i} & d_{\text {low }, i} \leq d \leq d_{\text {high }} \\
f_{\text {sat }} & 0 \leq d<d_{\text {low }, i}\end{cases} \\
A_{i}=g_{A}(d)= \begin{cases}k_{A, i} d+b_{A, i} & d_{\text {low }, i} \leq d \leq d_{\text {high }} \\
A_{\text {sat }} & 0 \leq d<d_{\text {low }, i}\end{cases}
\end{gathered}
$$

where $d$ represents the input drive signal received by the robot, which can be subdivided into the left and right drive signals $d_{L}$ and $d_{R}$, respectively. Individually, they drive the bilateral neural oscillators in the robot. $f_{\text {sat }}$ and $A_{\text {sat }}$ are the saturation values. $k_{f, i}, b_{f, i}, k_{A, i}, b_{A, i}$ are frequency coefficients and amplitude coefficients, which decide the evolution of the intrinsic frequency and amplitude of oscillator $i . d_{l o w, i}$ and $d_{\text {high }}$ correspond to the lower and upper thresholds separately. For each oscillator $i$, when the drive reaches the corresponding lower threshold $d_{l o w, i}$, the homologous oscillations start. For simplicity, the same values are set to a single type of parameter for symmetric oscillators located on both sides of the robot. The intrinsic frequencies and amplitudes of the oscillators increase until the upper threshold $d_{\text {high }}$ is attained, in which case the oscillators saturate and their frequencies and amplitudes maintain invariable. By modulating the lower threshold $d_{\text {low }, i}$, the corresponding joint can be enabled or not, which can realize various propulsion types coordinated by multiple propulsive elements. These are

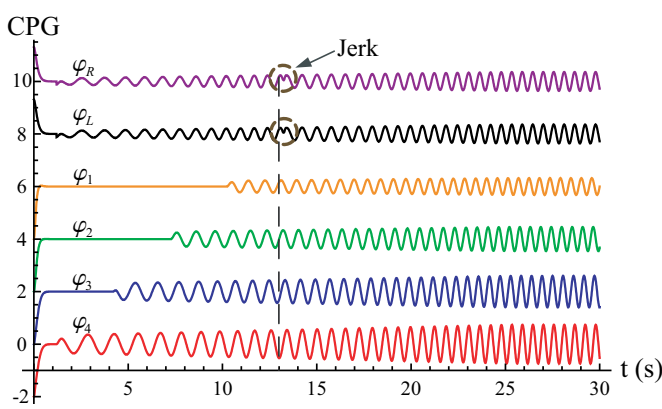

(a)

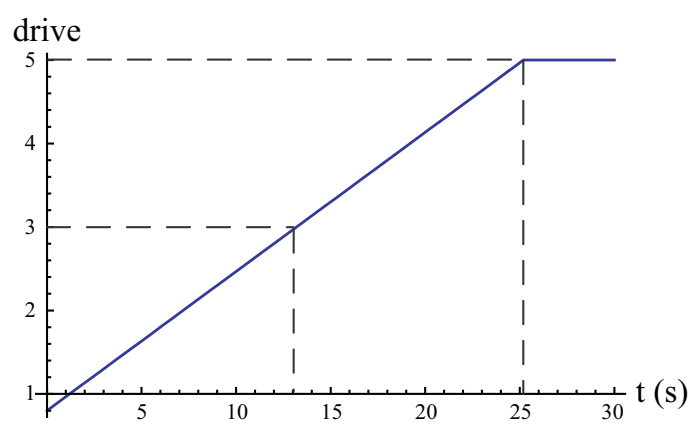

(b)

Fig. 7. Activity of the CPG model when the input drive is progressively increased. (a) Actuated joint angle signals from the CPG model. (b) Linear increase of input drive applied to the CPG model.

the same for the pectoral fin, which is denoted by $d_{\text {low,pec }}$. All of the above are the undecided coefficients of the CPG model.

For the convenience of control, all the oscillators of the tail CPG share the same frequency with different amplitudes in order to maintain the consistency of the body undulation. When asymmetrical drives are applied to the lateral turning locomotion, the drive signal takes the average of the ambilateral drives in (4). Oscillators in each joint possess the same amplitude coefficients while different coefficients are allotted to different joints. The pectoral fins in the bilateral positions of the head adopt the same frequency and amplitude coefficients, but different from the ones used in the body. The left and right drives are applied to the corresponding pectoral fins individually, which can contribute to turning when asymmetrical drives are applied.

In addition, fish rely on the individual oscillation of pectoral fins to propel itself at a low speed, or coordinated smallamplitude oscillation with the caudal fin at whiles. Once acceleration, the pectoral fins will oscillate in synchrony with the body to generate propulsion. From an engineering point of view, the tail CPG should inhibit the pectoral CPG unidirectionally at a given input drive. In such a case, an effective drive is introduced, defined as $d_{\text {effect }}=\left(d_{L}+d_{R}\right) / 2 \triangleq 3$. When the drive increases and the effective drive satisfies the condition, the tail CPG begins to inhibit the pectoral CPG and the strong coupling forces the pectoral fins to oscillate at the frequency of the body despite their own intrinsic frequencies. In the meanwhile, there will be a certain phase lag between neighboring oscillators based on the principle 


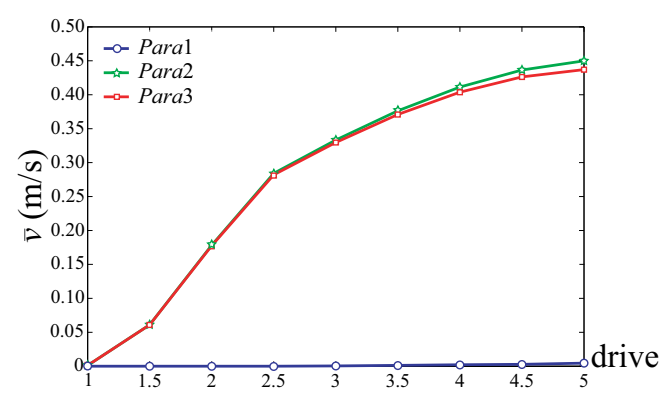

Fig. 8. Three types of forward gaits by modulating the threshold, where Para $1=\left\{d_{\text {low }, \text { pec }}=1.0, d_{\text {low }, i}=5.01(i=1, \ldots, 4), d_{\text {high }}=5.0\right\}$, Para $2=\left\{d_{\text {low pec }}=5.01, d_{\text {low }, 1}=2.5, d_{\text {low } 2}=2.0, d_{\text {low }, 3}=1.5, d_{\text {low }, 4}=\right.$ $\left.1.0, d_{\text {high }}=5.0\right\}$, Para $3=\left\{d_{\text {low }, \text { pec }}=1.0, d_{\text {low }, 1}=2.5, d_{\text {low }, 2}=2.0, d_{\text {low }, 3}=\right.$ $\left.1.5, d_{\text {low }, 4}=1.0, d_{\text {high }}=5.0\right\}$, as illustrated in Fig. 6 .

of body wave propagated from head to tail. Moreover, the phase lag between the pectoral CPG and tail CPG and their intersegmental couplings should also be considered which will fundamentally influence the activity of the CPG model. For more details of the couplings within the CPG model, refer to [11].

\section{C. $C P G$ Parameter}

One key property of the CPG is its adaptability to parameter changes. Despite abrupt parameter changes, the produced trajectory will smoothly converge toward the new limit cycle after a short transient period. When the above CPG model is used for the underwater swimming motion control of the AmphiRobot, several explicit parameters defining quantities should be determined, such as the time constant, threshold, frequency and amplitude coefficients, etc. These parameters can be continuously modified to produce adaptable gaits. Different control parameters will produce different CPG activities, with the alteration of joint angle control, which will ultimately influence the motion mode, swimming speed, propulsion efficiency, and movement stability, etc. Due to the complexity, nonlinear and strong coupling of the CPG model, purely analytical means are weak. The optimization of CPG parameters remains an urgent task to follow up. The trialand-error method based on simulation technology is often utilized to modulate the parameters, enabling the activity of the CPG model to approach the actual swimming gait, meeting the requirements of control tasks. Furthermore, the exploited high-performance microprocessor ensures a light computation of the CPG model which enables it suited to be programmed on board of the robot.

In practice, a control platform is developed which enables one to easily adjust the parameters of the CPG model, such as the frequency coefficients, amplitude coefficients, thresholds and input drives, as depicted in Fig. 6. Using this platform, the CPG parameters can be continuously and interactively modulated by a human operator to offer high maneuverability. These parameters have been kept into a reasonable range.

Fig. 7 depicts the activity of the CPG model according to the above parameters set when a linear increasingly drive

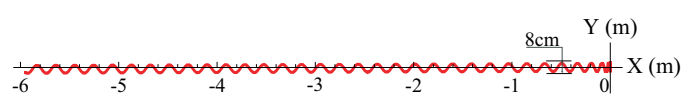

(a)

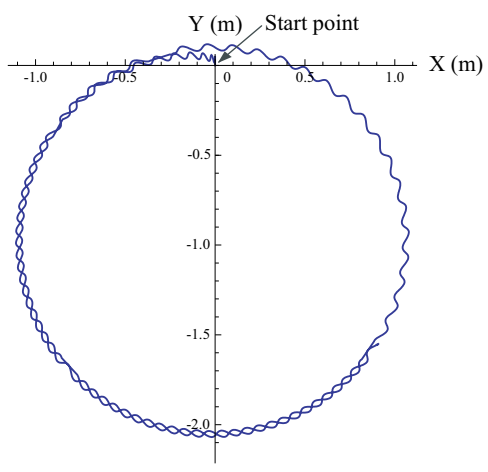

(b)

Fig. 9. Simulated trajectory with the parameters illustrated in Fig. 6 except the input drive. (a) Simulated forward swimming with $d_{L}=d_{R}=4$. (b) Simulated turning with $d_{L}=1.5, d_{R}=3.5$.

is applied in Fig. 7(b) (with the same drive on both sides, i.e. $d_{L}=d_{R}$ ). Thanks to the anti-interference characteristic of the CPG model, the initial values of the system (3) are any random numbers. Fig. 7(a) demonstrates that the system converges to the stable oscillations after a short time for a random perturbation. The oscillating joints gradually participate in the oscillations with the drive, from J4 to J1. At about $13 \mathrm{~s}$, an irregular jerk emerges for the activity of pectoral fins as the drive reaches the effective drive $d_{\text {effect }}$, and then the tail CPG begins to inhibit the pectoral CPG which is forced to oscillate at the frequency of the tail CPG for coordination. The jerk disappears quickly when the initial values converge to the regular traveling waves.

\section{Simulation And Experiments}

\section{A. Simulation}

To test the adopted CPG model systematically, some simulations have been conducted to demonstrate how the CPG parameters influence the locomotion speed. All the systematic tests have been conducted with one single parameter changed to be evaluated, while all other parameters are fixed to suitable values as illustrated in Fig. 6 .

Input drive is the parameter whose influence on the locomotion speed is the simplest: with all other parameters fixed (i.e. frequency coefficient, amplitude coefficient, and threshold), increasing the input drive generally leads to an increase of the speed. This facilitates the modulation of locomotion speed. Fig. 8 demonstrates interesting properties of the CPG model for parameter modifications. When modulating the threshold of each oscillating element, the robot can acquire different forward gaits by oscillating the body and/or caudal fin (BCF, with Para1 adopted), or pectoral fins (PF, with Para 2 adopted), or their combinations (with Para 3 adopted). The simulated speed partly confirms biologyinspired concept that fish typically rely on BCF mode for propulsion while employing PF mode for maneuvering and 


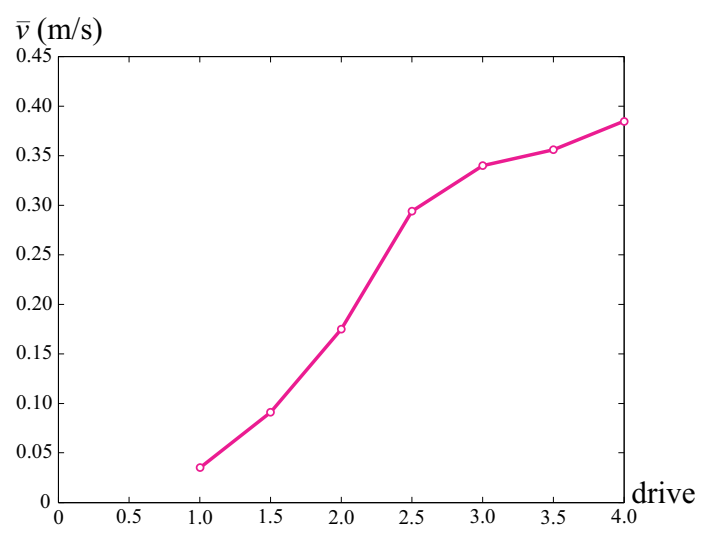

Fig. 10. Forward speed versus input drive.

stabilization. Further evaluations of the CPG parameters will be a foreseeable bonus.

As depicted by Fig. 9, simulated trajectories of forward gait and turning locomotion can be obtained under the parameter set in Fig. 6.

\section{B. Experiments}

1) Forward swimming: By applying the same drive to the left and right sides of the CPG model, the AmphiRobot will realize forward swimming. As the same drive is increasing and applied to both sides, the swimming distances and corresponding consuming time are measured at drives ranging from 1.0 to 4.0 with a step of 0.5 . The measurement has been run three times for a specific drive. The average speed can thus be calculated.

Fig. 10 demonstrates how the input drive affects the propulsion speed with all oscillating elements gradually involved in the locomotion under the parameter set in Fig. 6. The speed increases with the drive and attains the maximum value when the input reaches the maximum acceptable drive.

2) Turning: Turning can be induced when one side of the robot receives more excitation than the other. The simplest approach is to apply a left-right asymmetry of input drive. The snapshots of the turning locomotion are plotted in Fig. 11 at $d_{L}=1.5, d_{R}=3.5$, and the average turning radius is around $0.8 \mathrm{~m}$, which is minor in contrast with Fig. 9(b). It is possibly owing to the unbalance of the robot's body which tilts to the side of smaller drive. The experimental errors should also be taken into consideration.

\section{COnClusions And Future Work}

This paper presents an amphibious robot capable of multimode motion controlled by a CPG model. The CPG model is designed to produce the traveling wave as the joint angle

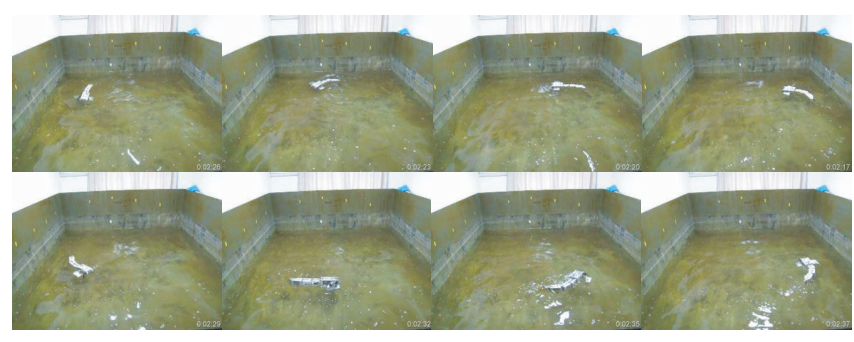

Fig. 11. The snapshots of the turning locomotion.

control of fish-like propelling units. By modulating the control parameters of the CPG model, various types of gaits can be obtained for steady swimming. The experiments are also conducted to testify the proposed CPG model.

The input drive of the CPG model is the simplest and most effective parameter for locomotion control. The oscillation frequencies and amplitudes depend on the input drive, as formulated in (4) and (5). The bridge connecting them is the parameters of the CPG model, as listed in Fig. 6, which need to be optimized. The future work will focus on the parameter optimization of the CPG model to adapt optimal gaits to various environments. More efforts will be paid to the intelligent switch of multi-mode motions and construction of a self-contained autonomous control system ultimately.

\section{REFERENCES}

[1] M. J. Lighthill, "Note on the swimming of slender fish, Part2," J. Fluid Mech., vol. 9, pp. 305-317, 1960.

[2] T. Y. Wu, "Swimming of a waving plate," J. Fluid Mech., vol. 10, pp. 321-344, 1961.

[3] M. G. Chopra and T. Kambe, "Hydromechanics of lunate-tail swimming propulsion, Part2," J. Fluid Mech., vol. 79, pp. 49-69, 1977.

[4] J. J. Videler and F. Hess, "Fast continuous swimming of two pelagic predators, saithe (pollachius virens) and mackerel (scomber scombrus): A kinematic analysis," J. Exp. Biol., no. 109, pp. 209-228, 1984.

[5] F. A. Mussa-Ivaldi and S. A. Solla, "Neural primitives for motion control," IEEE J. Ocean. Eng., vol. 29, no. 3, pp. 640-650, 2004.

[6] A. Crespi, A. Badertscher, A. Guignard, and A. J. Ijspeert, "AmphiBot I: An amphibious snake-like robot," Robot. Auton. Syst., vol. 50, pp. 163-175, 2005.

[7] A. Crespi and A. J. Ijspeert, "AmphiBot II: An amphibious snake robot that crawls and swims using a Central Patten Generator," in Proc. 9th Int. Conf. Climbing Walking Robots, 2006, pp. 19-27.

[8] S. Grillner, P. Wallen, and L. Brodin, "Neuronal network generating locomotor behavior in lamprey: Circuitry, transmitters, membrane properties, and simulation," Annu. Rev. Neuroscience, vol. 14, pp. 169199, 1991.

[9] A. J. Ijspeert, A. Crespi, D. Ryczko, and J-M. Cabelguen, "From swimming to walking with a salamander robot driven by a spinal cord model," Sci., pp. 1416-1420, 2007.

[10] R. Ding, J. Yu, Q. Yang, X. Hu, and M. Tan, "Platform-level design for a biomimetic amphibious robot," in Proc. IEEE Int. Conf. Robot. Biomim., Bangkok, Thailand, Feb. 21-26, 2009, pp. 977-982.

[11] Q. Yang, "Design and control of a biomimetic amphibious robot with multi-mode motion," Doctoral dissertation, Beijing: Institute of Automation, Chinese Academy of Sciences, 2009 (in Chinese). 